\title{
Synthesis of Flexible Accelerators for Early Adoption of Ring-LWE Post-quantum Cryptography
}

\author{
HAMID NEJATOLLAHI, University of California Irvine \\ FELIPE VALENCIA, ALaRI \\ SUBHADEEP BANIK, EPFL \\ FRANCESCO REGAZZONI, ALaRi \\ ROSARIO CAMMAROTA, Intel AI Research \\ NIKIL DUTT, University of California Irvine
}

The advent of the quantum computer makes current public-key infrastructure insecure. Cryptography community is addressing this problem by designing, efficiently implementing, and evaluating novel public-key algorithms capable of withstanding quantum computational power. Governmental agencies, such as NIST, are promoting standardization of quantum-resistant algorithms that is expected to run for 7 years. Several modern applications must maintain permanent data secrecy; therefore, they ultimately require the use of quantum-resistant algorithms. Because algorithms are still under scrutiny for eventual standardization, the deployment of the hardware implementation of quantum-resistant algorithms is still in early stages.

In this article, we propose a methodology to design programmable hardware accelerators for lattice-based algorithms, and we use the proposed methodology to implement flexible and energy efficient post-quantum cache-based accelerators for NewHope, Kyber, Dilithium, Key Consensus from Lattice (KCL), and R.EMBLEM submissions to the NIST standardization contest.

To the best of our knowledge, we propose the first efficient domain-specific, programmable cache-based accelerators for lattice-based algorithms. We design a single accelerator for a common kernel among various schemes with different kernel sizes, i.e., loop count, and data types. This is in contrast to the traditional approach of designing one special purpose accelerators for each scheme.

We validate our methodology by integrating our accelerators into an HLS-based SoC infrastructure based on the X86 processor and evaluate overall performance. Our experiments demonstrate the suitability of the approach and allow us to collect insightful information about the performance bottlenecks and the energy efficiency of the explored algorithms. Our results provide guidelines for hardware designers, highlighting the optimization points to address for achieving the highest energy minimization and performance increase. At the same time, our proposed design allows us to specify and execute new variants of lattice-based schemes with superior energy efficiency compared to the main application processor without changing the hardware acceleration platform. For example, we manage to reduce the energy consumption up to $2.1 \times$ and energydelay product (EDP) up to $5.2 \times$ and improve the speedup up to $2.5 \times$.

\footnotetext{
This work was supported in part with a gift from Qualcomm Technology Inc., Swiss National Science Foundation project No P1TIP2_181305 and European Union Horizon 2020 research and innovation programme under SAFEcrypto project (grant agreement No 644729). Additionally, Subhadeep Banik was supported by Swiss National Science Foundation project No PZ00P2_179921.

Authors' addresses: H. Nejatollahi and N. Dutt, University of California Irvine, Irvine, California, 92697-3435; emails: hnejatol@uci.edu, dutt@ics.uci.edu; F. Valencia and F. Regazzoni, ALaRI; emails: valena@usi.ch, regazzoni@alari.ch; S. Banik, EPFL; email: subhadeep.banik@epfl.ch; R. Cammarota, Intel AI Research; email: rosario.cammarota@intel.com.

Permission to make digital or hard copies of all or part of this work for personal or classroom use is granted without fee provided that copies are not made or distributed for profit or commercial advantage and that copies bear this notice and the full citation on the first page. Copyrights for components of this work owned by others than ACM must be honored. Abstracting with credit is permitted. To copy otherwise, or republish, to post on servers or to redistribute to lists, requires prior specific permission and/or a fee. Request permissions from permissions@acm.org. (C) 2020 Association for Computing Machinery.

1539-9087/2020/03-ART11 \$15.00

https://doi.org/10.1145/3378164
} 
CCS Concepts: • Security and privacy $\rightarrow$ Public key (asymmetric) techniques; Hardware-based security protocols;

Additional Key Words and Phrases: Public key cryptography, post-quantum cryptography lattice-based cryptography, ideal lattices, domain specific acceleration, cache architecture

\section{ACM Reference format:}

Hamid Nejatollahi, Felipe Valencia, Subhadeep Banik, Francesco Regazzoni, Rosario Cammarota, and Nikil Dutt. 2020. Synthesis of Flexible Accelerators for Early Adoption of Ring-LWE Post-quantum Cryptography. ACM Trans. Embed. Comput. Syst. 19, 2, Article 11 (March 2020), 17 pages.

https://doi.org/10.1145/3378164

\section{INTRODUCTION}

A worldwide research effort is currently pursuing a scalable quantum computer. Quantum computers, as envisioned by Richard Feynman [14], use quantum mechanics to carry out computation that can reach an exponential speedup in several applications, such as quantum chemistry [29]. Exponential speed up can also be reached in other problems, such as the search for the period of a function. As demonstrated by Peter Shor [34], it would allow us to factorize integers in polynomial time and ultimately render the public key infrastructure insecure. Post-quantum cryptography is a vibrant area of research devoted to studying alternative public-key algorithms, executed on classical computers but capable of withstanding quantum computational power. The effort of governmental agencies in conducting the standardization of these novel algorithms endorses the urgency of the threat. The most relevant standardization effort is that of NIST, which started in 2017 with the submissions of potential candidates and continues with the evaluation of the candidates for the next 6 to 7 years, until the selection of the new standards.

In the long run, achieving resistance against quantum attacks is vital, because there will be devices based on classical architectures even after the invention of quantum computers. Quantum resistance is fundamental for an application that requires the "long-term security," namely that the data should be kept secret for an extended time-period (even much longer than the one envisioned for building a quantum computer). For such applications, it is of utmost importance to have an immediate deployment of quantum-resistant algorithms.

The experimentation of quantum-resistant algorithms is already ongoing in software platforms (for instance, Google Chrome started the tests of NewHope). However, for the complete adoption of post-quantum algorithms, it is necessary to meet the requirements of specific applications that necessarily need to rely on dedicated hardware. The early adoption of quantum-resistant hardware is challenging, because in this period of transition, during which candidates are still under the evaluation, a post-quantum standard is yet to be decided and also the parameters of the candidates themselves can change; subsequently, it is troublesome to commit to a specific algorithm and a specific set of parameters, knowing that they could be soon outdated [27].

The situation would change if the accelerators would be able to meet two requirements: (1) reach the required performance and (2) be flexible, thus "robust" to change in parameters and algorithms (providing the so-called crypto-agility). To meet these goals, we propose to accelerate, instead of a complete algorithm, only several portions of the software routines, called micro-kernels. Compared to a whole dedicated accelerator, a micro-kernel accelerator does not reach the same level of performance, because the main processor executes parts of the computation. Subsequently, hardware acceleration based on micro-kernels is more flexible compared to the classical counterparts. We provide two dimensions of flexibility by designing a single 32-bit accelerator for a kernel that is used by various schemes with different data types, e.g., uint32_t, uint16_t, int32_t, int16_t. The 
size of the same kernel can be different over different schemes, e.g., ranging from 128 to 512 loop iterations. We design a single accelerator by applying different compiler and architectural optimizations that can execute the same kernel for all the schemes.

To enable early adoption of quantum-resistant algorithms, in this article, we use a design space exploration framework, adopted by us to post-quantum cryptography, to explore the design space of micro-kernel-based acceleration of lattice-based cryptography (LBC) algorithms and to implement them. We focus on domain-specific programmable hardware accelerators, because they are the most suitable, at this stage, to adopt post-quantum algorithms in hardware. We concentrate on lattice-based algorithms, the most promising family of quantum-resistant algorithms thanks to their versatility and their excellent performance. Around half of the submission to the first round of the NIST PQC Standardization Process belong to this family. After a year of evaluation, 26 of the candidates are chosen for the second round; twelve candidates belong to lattices, and the remaining 14 candidates belong to code-based, hash-based, supersingular elliptic curve isogeny, and multivariate cryptography [27]. Finally, we concentrate on energy and performance as the main figure of merit, since these would be the most relevant parameters for IoT and battery-operated devices, as well as for servers and cloud accelerators [23].

The rest of the article is organized as follows: Section 2 provides the background for LBC primitives and summarizes the state of the art in design space exploration for cryptography and in accelerating LBC; Section 3 and Section 4 describe the design flow steps and the profiling results for the selected algorithms. Section 5 discusses the DSE results and elaborates on the design point selection.

\section{BACKGROUND AND RELATED WORKS}

A lattice $L \subset \mathbb{R}^{n}$ is the set of all integer linear combinations of basis vectors $\mathbf{b}_{1}, \ldots, \mathbf{b}_{n} \in \mathbb{R}^{n}$. i.e., $L=\left\{\sum a_{i} \mathbf{b}_{i}: a_{i} \in \mathbb{Z}\right\}$. In particular, $L$ is a subgroup of $\mathbb{R}^{n}$ that is isomorphic to $\mathbb{Z}^{n}$. The cryptography based on lattices exploits the hardness of two problems: Short Integer Solution (SIS) and Learning With Errors (LWE). Cryptosystems based on the LWE problem, the most used one, have their foundation in the difficulty of finding the secret key $s k$, given $(A, p k)$, where $p k=A * s k+e \bmod q$, with $p k$ being the public key, $e$ an error vector with Gaussian distribution, and $A$ a matrix of constants in $\mathbb{Z}_{q}^{r \times n}$ chosen randomly from a uniform distribution. In general, the parameters $n, r$, and $q$ are integers such that $n>1, r>n$, and $q \geq 2$, but for some efficient implementations these numbers are constrained to a limited set of values. For more detailed information about the parameters, we refer to the survey done by Oded Regev [31].

LWE, however, requires large keys that could be impractical; for instance, public and secret key size of Frodo [22], LWE-based KEM, at NIST security level 1 are $9 \mathrm{~KB}$ and $19.8 \mathrm{~KB}$, respectively. To overcome this limitation, Lyubashevsky et al. [21] introduced Ring-LWE (RLWE), a derivation of LWE where $A$ is implicitly defined as a vector $a$ in the $\operatorname{ring} \mathcal{R} \equiv \mathbb{Z}_{q}[x] /\left\langle x^{n}+1\right\rangle$. These lattices are called Ideal lattices, while other lattices are called Standard lattices. At the same security level as Frodo, NewHope [30], a RLWE-based KEM, provides 10× smaller public and secret keys. Since implementing a polynomial multiplication requires a notable amount of computational resources, it is implemented using the so-called Number Theoretic Transform (NTT), which is a Fast Fourier Transform implemented over a ring. In the rest of this section, we introduce a detailed definition of RLWE and number-theoretic transform.

\section{Notations}

In an RLWE encryption scheme, operations are performed on polynomials. We denote polynomials in the time domain by a lowercase letter, e.g., $x$, and whenever we want to access one coefficient of the polynomial, we denote $x(i)$. Polynomials in the frequency domain are denoted by uppercase 

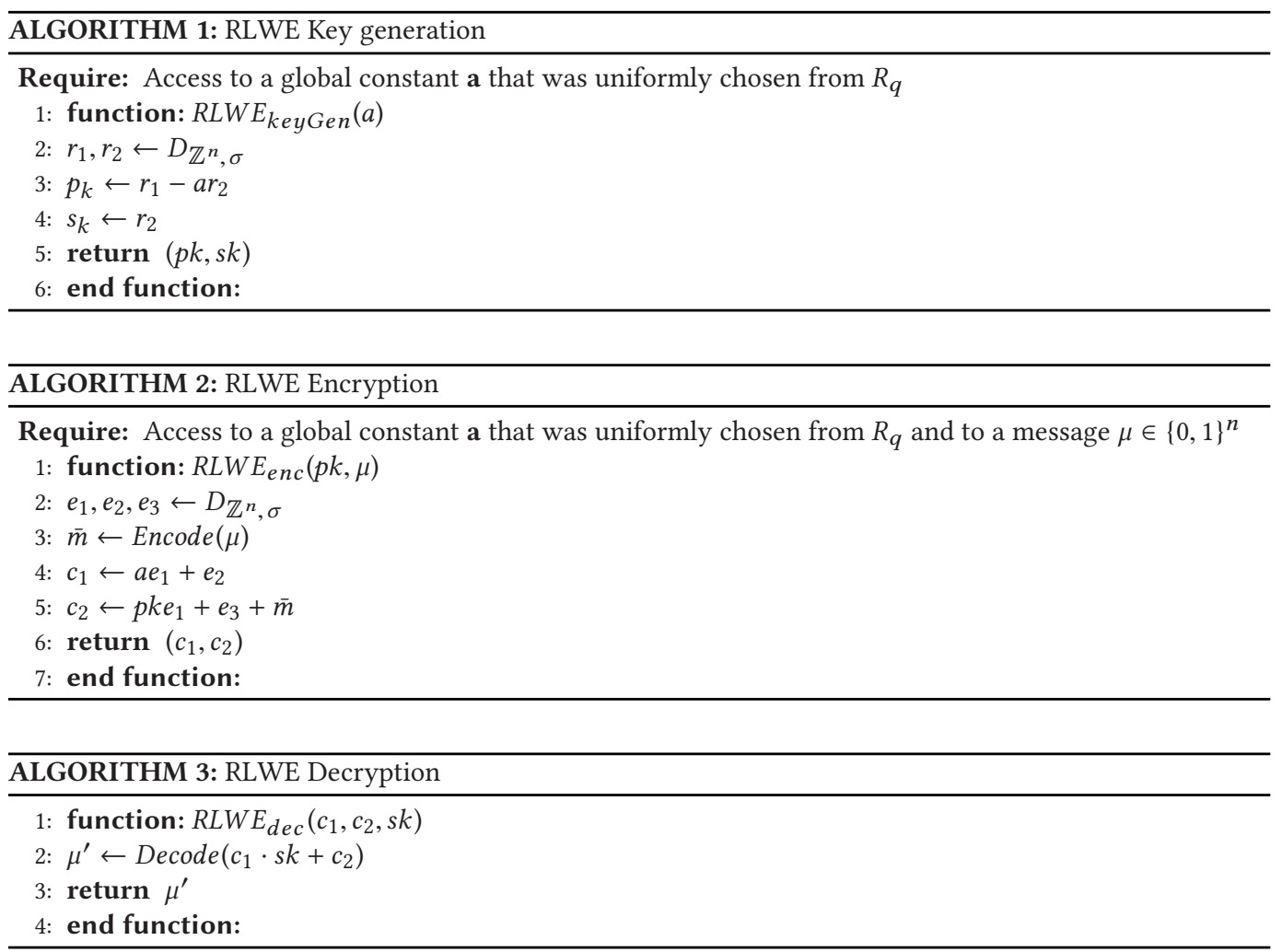

letters. Multiplication of integers is described by $\cdot$ while we use $*$ for multiplication of polynomials. We omit · and $*$ where it is clear from the context.

\subsection{Ring-LWE Encryption Scheme}

The cryptosystems analyzed in this article are mostly based on a ring-variant of the LWE problem $[21,31]$. The hard problem to be solved on the $\operatorname{ring} \mathcal{R}=\mathbb{Z}_{q}[\mathbf{x}] /\left\langle x^{n}+1\right\rangle$, which defines as deciding whether the samples $\left(a_{1}, t_{1}\right), \ldots,\left(a_{m}, t_{m}\right) \in \mathcal{R} \times \mathcal{R}$ are chosen uniformly random or whether each $t_{i}=a_{i} s+e_{i}$, being $s, e_{1}, \ldots, e_{m}$ small coefficients from the (one-dimensional) discrete Gaussian distribution $\mathcal{D}_{\sigma}$ [21]. The distribution $\mathcal{D}_{\sigma}$ is defined such that a value $x \in \mathbb{Z}$ is sampled from $\mathcal{D}_{\sigma}$ with the probability $\rho_{\sigma}(x) / \rho_{\sigma}(\mathbb{Z})$, where $\rho_{\sigma}(x)=\exp \left(\frac{-x^{2}}{2 \sigma^{2}}\right)$ and $\rho_{\sigma}(\mathbb{Z})=\sum_{k=-\infty}^{\infty} \rho_{\sigma}(k)$.

The algorithms for key generation, encryption, and decryption are presented in Algorithm 1 , Algorithm 2, and Algorithm 3, respectively. The key generation uses a constant vector $a$ for obtaining the public and secret key $(p k, s k)$. The encryption procedure takes a binary string $\mu$ and releases the ciphers $\left(c_{1}, c_{2}\right)$, which are used in the decryption to recover the message $\mu^{\prime}$. The vectors $r_{1}, r_{2}, e_{1}, e_{2}, e_{3}$ are error vectors sampled from a Gaussian distribution.

The Number-Theoretic Transform (NTT) transforms a finite field polynomial from the time to the frequency domain. Inverse Number-Theoretic Transform $\left(N T T^{-1}\right)$ transforms back the polynomial to the time domain. Polynomial multiplication in the frequency domain has linear complexity instead of quadratic complexity. As the transformation itself has quasi-linear complexity, the overall effort for polynomial multiplication drops from $O\left(n^{2}\right)$ to $O(n \cdot \log n)$. The efficient computation of the NTT involves the coefficient ring to contain primitive roots of unity. Formally, a primitive root of unity is defined as follows. 
Definition 2.1 (Primitive Root of Unity). Let $\mathcal{R}$ be a ring, $n \in \mathbb{N}_{\geq 1}$, and $\omega \in \mathcal{R}$. The value $\omega$ is an $n$th root of unity if $\omega^{n}=1$. The value $\omega$ is a primitive $n$th root of unity (or root of unity of order $n$ ) if it is an $n$th root of unity, $\omega^{n} \in \mathcal{R}$ is a unity in $\mathcal{R}$, and $\omega^{n / t}-1$ is not a zero divisor for any prime divisor $t$ of $n$.

For a given primitive $n$th root of unity $\omega$ in $\mathbb{Z}_{q}$, the NTT of a vector $a=(a(n-1), \ldots, a(0))$ is the vector $A=(A(n-1), \ldots, A(0))$ and it is computed as $A(i)=\sum_{j=0}^{n-1} a(j) \omega^{i j} \bmod q, i=0,1, \ldots, n-$ 1. The inverse transformation is calculated as $a(i)=n^{-1} \sum_{j=0}^{n-1} A(j) \omega^{-i j} \bmod q, i=0,1, \ldots, n-1$.

The idea is to transform two polynomials $a=a(n-1) \cdot x^{n-1}+\cdots+a(0)$ and $b=b(n-1)$. $x^{n-1}+\cdots+b(0)$ into their NTT representations $A=A(n-1) \cdot x^{n-1}+\cdots+A(0)$ and $B=B(n-$ 1) $\cdot x^{n-1}+\cdots+B(0)$ and to compute the coefficient-wise multiplication as $C=\sum_{i=0}^{n-1} A(i) \cdot B(i)$. $x^{i}$. The result $c=a * b$ is obtained after the computation of the inverse number theoretic transform $\left(N T T^{-1}\right)$ to $C$. For $q=1 \bmod 2 n$, the way the result has to be interpreted depends on the input. Two common algorithms of performing number theoretic transform are Cooley-Tukey (CT) [10], which produces the result in the bit-reverse order by receiving the input in the correct order, and Gentleman-Sande (GS) [16], which receives the input in the reverse order and produces the output in the correct order. We refer to Gentleman-Sande and Cooley-Tukey algorithms as NTT_CT and NTT_GS, respectively, in the rest of this article. Employing NTT_GS to compute both NTT and $N T T^{-1}$ involves in bit-reverse calculation (NewHope); however, bit-reverse step can be skipped by using NTT_CT for NTT and NTT_GS for NTT ${ }^{-1}$ (Kyber and Dilithium).

Keccak is a family of cryptographic primitives primarily as a candidate for the NIST SHA-3 competition [8]. The main transformation in the family is the Keccak-f[1600] permutation, which is used in the sponge mode in the specification for the SHA-3 hash function. The hash function operates on a state of 1,600 bits and is designed for easy implementation in both software and hardware. The state can be considered to be a $5 \times 5 \times 64$ array of bits. Let $a[i][j][k]$ be bit ( $5 i+$ $j) \times w+k$ of the input, using a little-endian bit numbering convention and row-major indexing. Index arithmetic is performed modulo 5 for the first two dimensions and modulo $w$ for the third. A set of 64 bits with the same $x, y$ coordinates is called a lane. The basic block permutation function consists of 24 rounds of five steps (Figure 7).

- $\theta$ : In this step, we compute the parity of each of the 320 five bit columns and add it two nearby columns. Functionally it can be described as $a[x][y][z] \leftarrow a[x][y][z]+$ $\sum_{y^{\prime}=0}^{4} a[x][y][z]+\sum_{y^{\prime}=0}^{4} a[x+1][y][z-1]$

- $\rho$ : In this step we rotate each of the lanes by a fixed number of bits. The transformation is given by $a[x][y][z] \leftarrow a[x][y][z-(t+1)(t+2) / 2]$ where $t=-1$, if $x=y=0$ and otherwise $t \in[0,24]$ satisfying $\left(\begin{array}{l}x \\ y\end{array}\right)=\left(\begin{array}{ll}3 & 2 \\ 1 & 0\end{array}\right)^{t}\left(\begin{array}{l}0 \\ 1\end{array}\right)$

- $\pi$ : The mapping $\pi$ is a transposition of the lanes. It is defined as $a[x][y][z] \leftarrow$ $a\left[x^{\prime}\right]\left[y^{\prime}\right][z]$, with $x=y^{\prime}, y=2 x^{\prime}+3 y^{\prime}$

- $\chi$ : This is the only non-linear step in the round function given by $a[x][y][z] \leftarrow a[x][y][z]+$ $(a[x+1][y][z]+1) a[x+2][y][z]$

- $l$ : This is the round constant addition step. A round constant is added only to the lane with co-ordinates $(0,0)$.

The energy cost of implementation is crucial for constraint devices and servers; energy consumption of classical cryptography has been evaluated by many authors [3, 11, 36, 37]. However, for lattice-based cryptography, previous works focused mainly on limiting the area and improving the performance $[5,17]$. However, energy has been left mostly unexplored, except for FPGA implementation [25], ASIC implementation [6], and simulation-based [26] accelerator for NTT, 


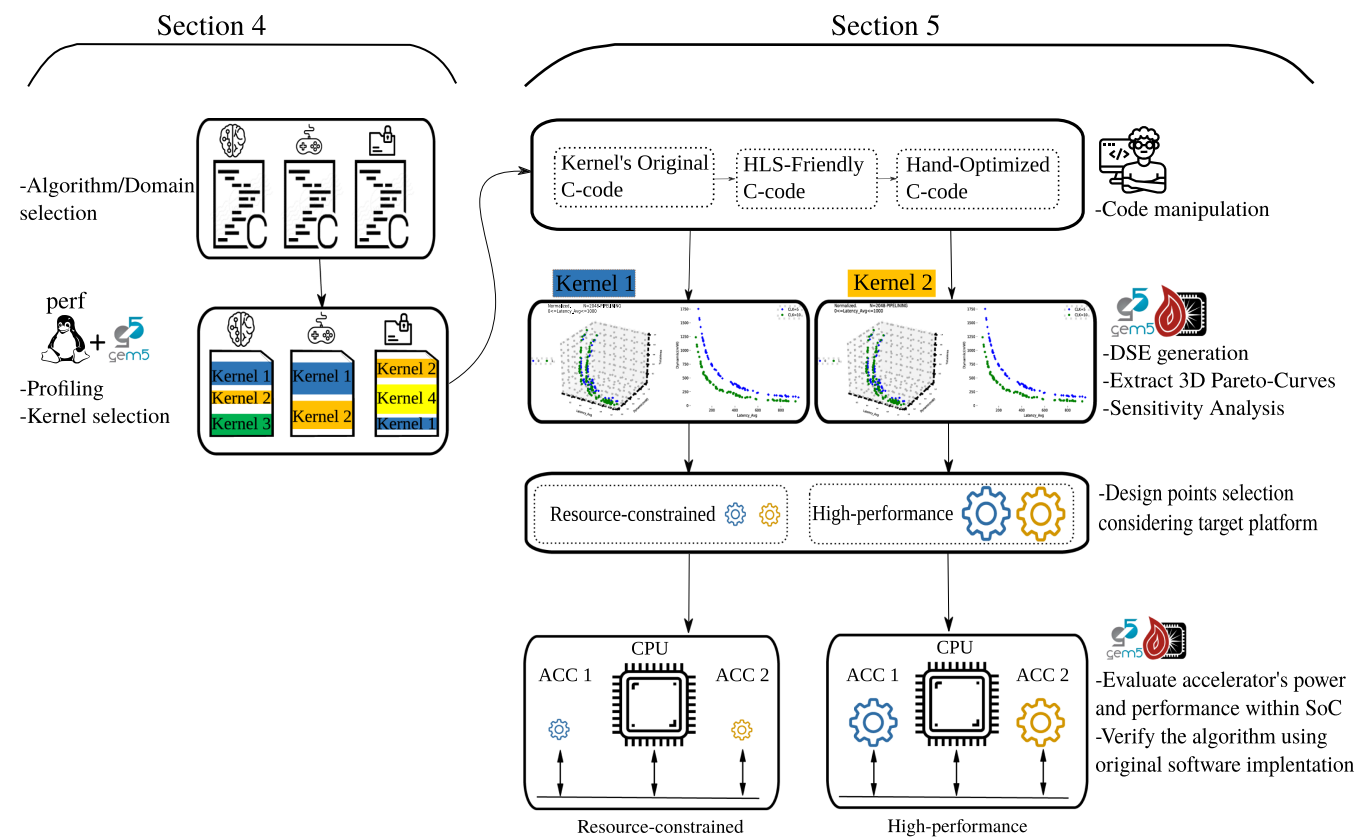

Fig. 1. Design flow of the accelerator generation. After choosing the domain and algorithms, we identify the common kernels by profiling the applications using perf Linux and gem5 [9]. The next steps are the code manipulation, DSE generation (using gem5-Aladdin), and sensitivity analysis. Afterward, we select the best design point for each kernel by considering the target platform. We evaluate the accelerator's power and performance within the SoC inside the gem5-Aladdin [33] simulator. The last step is to verify the algorithm using the original software implementation.

where none of them are cache-based accelerators. The early results of this work were presented in Reference [24].

\section{DESIGN FLOW STEPS}

In this section, we detail the three main steps that compose our design space exploration flow: profiling, early exploration, and evaluation within the target architecture. Figure 1 illustrates the design flow steps to generate flexible accelerators for any domain or set of algorithms. The first step is the profiling of the implementations of the target lattice-based schemes. This process is needed to identify the bottlenecks and the kernel to be accelerated. Profiling is executed by running the algorithm on a host arbitrary machine, e.g., X86-based system, and verify it using a microarchitectural simulator such as gem5 [9]. In the former case, profiling results correspond to collecting performance counters from the execution. In the latter case, profiling means collecting simulation statistics. In either case, the same hot-spots in the protocol are identified. We profile the server and the client portion of the protocol separately to accommodate the specific needs of each endpoint. The identified hot-spot functions are candidates for acceleration and are input in our design exploration flow.

In the second step, we used gem5-Aladdin [33] to perform the design space exploration of the candidate functions. gem5-Aladdin is a fast design exploration framework that allows making decisions on the tradeoff between performance, power, and area of the identified design points with high accuracy, even avoiding the explicit HLD synthesis step. For standard linear algebra kernels, pre-RTL performance area and power estimation fall within a threshold of $10 \%$ compared 
to the actual HLD synthesis.o The results of the DSE are stored in an elastic search service, which we develop to keep track of the design points and to compare them in terms of area, power, performance, and complexity. The DSE itself consists on the extraction of instruction-level parallelism in each candidate function and the mapping of such parallelism on a parameterizable set of finite constraints in the hardware, e.g., number of vector lanes, vector lane pipeline depth, ports to memory, and communication to memory (with cache or via streaming) [33]. The introduction of system-level requirements allows us to query the elastic search service and to prune down the set of possible designs further. We use three-dimensional (latency, power, and area) Pareto-optimal fronts to eliminate non-optimal points. The design points that are not pruned will be evaluated in the whole system on the chip.

In the third step, we exploit the integration between gem5 and Aladdin [33] to evaluate the accelerator within the whole SoC. We used this approach to avoid Transaction Level Simulation, emulation, or full-system HLD synthesis. The memory communication and the host processor computation aspects are modeled in a pre-RTL environment. Thanks to the accuracy guaranteed by gem 5 and Aladdin [33], this last step allows us to identify a restricted set of design points that quickly reach our design goals.

In this work, we accelerate the execution of lattice-based cryptography algorithms using coprocessors with the small instruction set. Based on Figure 7, these accelerators are loosely coupled with the main processor in a System on Chip (SoC). Each accelerator has its private caches and operates in slave mode attached to the network o chip.

The accelerators are kernel-level accelerators, where the kernel to accelerate is the bottleneck functions that are common across different families of lattice-based cryptography schemes. For instance, any call to NTT_GS is transferred to the accelerator; if the size of the NTT_GS, i.e., polynomial degree, which determines the security strength, is bigger than the accelerator, we orchestrate the data stream via the cache hierarchy. In other words, the accelerator is a pipelined implementation of the kernel that arbitrary size of a call to the kernel can be offloaded to it.

The choice of this type of accelerator allows us to maintain high flexibility while at the same time, improving performance. This strategy ensures crypto-agility, which is fundamental for the early adoption of post-quantum algorithms. Additionally, it allows us to accelerate several latticebased cryptography schemes with a small set of co-processors. Both advantages could not have been achieved using designs customized on each scheme.

\section{ALGORITHMS PROFILING}

We considered four key encapsulation mechanisms (NewHope, Kyber, R.EMBLEM, and KCL) and a digital signature algorithm (Dilithium). A KEM is a technique to encapsulate a symmetric key using an asymmetric cryptographic algorithm [28]. A digital signature is a scheme that guarantees the integrity and authenticity of a message. In the rest of the section, we discuss their profiling results.

\subsection{NewHope}

NewHope cryptosystem is a suite of two KEMs based on NewHope-Simple [2] that bases its security on the hardness of RLWE problem. NewHope is the transformation of a Public Key Encryption (PKE) scheme into a KEM using the Targhi and Unruh transforms [35] that implies the use of hash functions, which in the case of NewHope is Shake256 [13]. Also, NTT and binomial samplers are employed to improve the performance of the algorithm. The current submission of NewHope to NIST competition presents two parameter sets, NewHope512cpa/cca and NewHope1024cpa/cca, with security levels of 101 bits and 233 bits, depending on the length of ring polynomials [30]. 

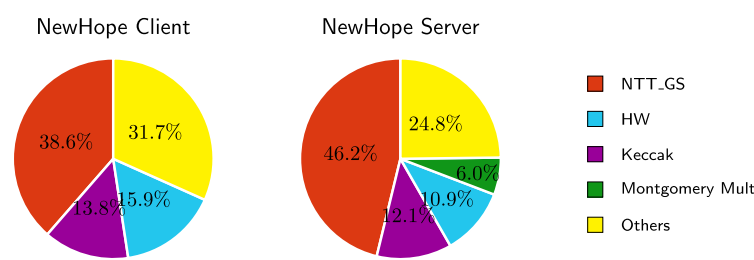

Fig. 2. Profiling results for NewHope: Client-Server. Profiling results are based on the user-level CPU cycles.
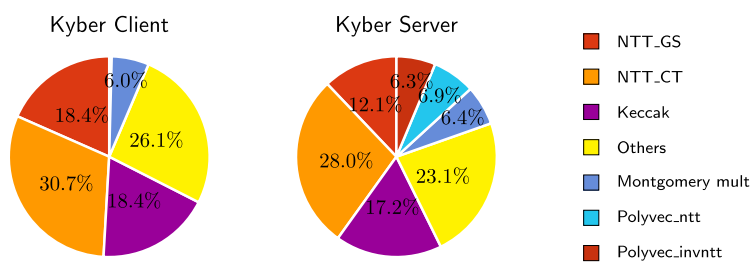

Fig. 3. Profiling results for Kyber: Client-Server. Profiling results are based on the user-level CPU cycles.
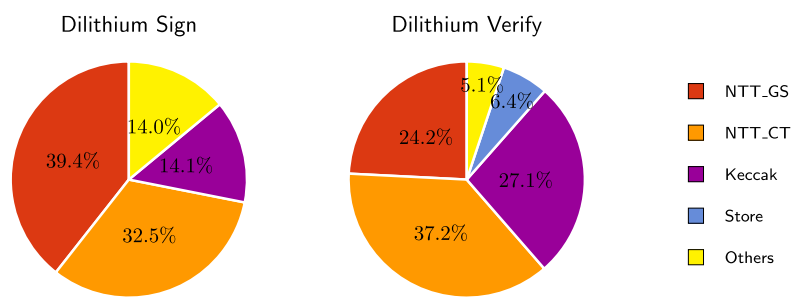

Fig. 4. Profiling results for Dilithium: Sign-Verify. Profiling results are based on the user-level CPU cycles.

Figure 2 presents the profile of NewHope1024cpa, referred to as NewHope in the rest of the article, in the client and server-side. NewHope employs NTT_GS for both forward and inverse NTT.

\subsection{Kyber}

Kyber is a KEM based on the LWE problem in module lattices [19] that is built using the FujisakiOkamoto transform [15]. Kyber uses NTT_CT and NTT_GS to compute forward and reverse NTT, respectively. Kyber is presented in three versions: Kyber512, Kyber768, and Kyber1024 with the quantum hardness 102, 161, and 241 bits, respectively [4]. Due to the similarity, we only analyze Kyber768 in this article. The profile of Kyber768, referred to as Kyber in the rest of the article, is shown in Figure 3.

\subsection{Dilithium}

Dilithium is a signature algorithm based on the "Fiat-Shamir with Aborts" approach [20] that is designed to be easy to implement securely [12]. Its main characteristics are small signature and keys, conservative parameters, and modular structure. The most relevant operations in Dilithium are eXtendable-Output Functions (XOF) and polynomial multiplication in a ring. SHAKE128 and Shake256 are used as XOF. Similarly to Kyber, Dilithium uses NTT_CT and NTT_GS to compute forward and reverse NTT, respectively. The profile of Dilithium for signature and verification processes are reported in Figure 4. 

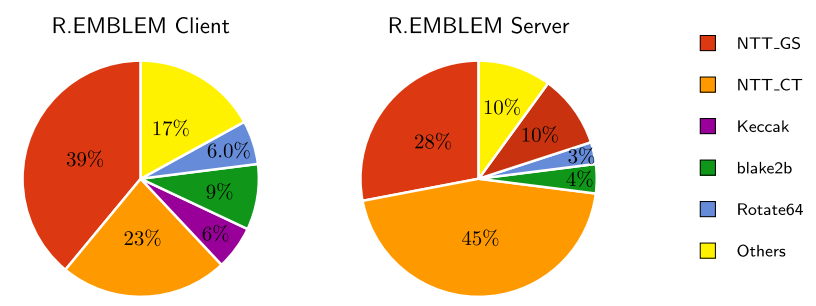

Fig. 5. Profile R.EMBLEM: Client-Server.
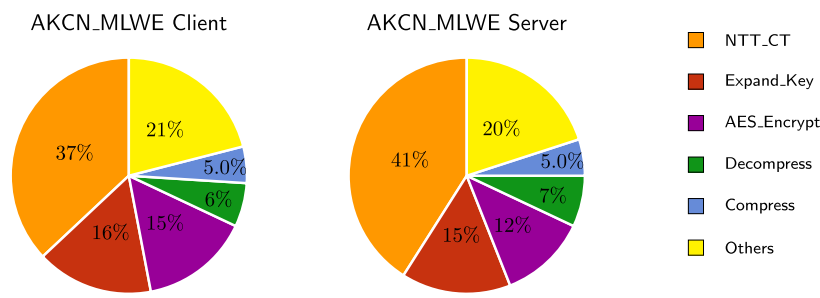

Fig. 6. Profile AKCN_MLWE: Client - Server.

\subsection{R.EMBLEM}

R.EMBLEM (and its standard lattice variant EMBLEM) is an error-blocked multi-bit RLWE-based (LWE-based) key encapsulation mechanism [32]. Small keys size is achieved by separating the message from error and attach the error-blocking bit to each message block. Sampling the secret term is performed uniformly at random in $[-B, B]$. Similarly to Kyber and Dilithium, R.EMBLEM uses NTT_CT and NTT_GS to compute forward and reverse NTT, respectively.

\subsection{KCL (Key Consensus from Lattice)}

Zhao et al. [38] propose a generic construction of the authenticated key exchange, public-key encryption, and key encapsulation mechanism, including asymmetric key consensus (AKCN) and optimal asymmetric key consensus (OKCN). Proposed schemes are based on LWE and its variants over ideal and module lattices with different mathematical structures. Authors propose single error correction (SEC) code to correct at least one-bit error; using the SEC, at the same security level and error rate, OKCN/AKCN-RLWE-based KEX schemes generate 765-bit shared a key with less bandwidth than NewHope (256-bit shared key). Due to the similarity and to avoid the repetition, we provide the results for AKCN-MLWE. AKCN-MLWE uses NTT_CT to compute the forward and reverse NTT.

Table 1 shows summarized the size of the common kernels and the data types that they perform the computations. Keccak performs 25 iterations on unsigned 64-bit data. However, for NTT_GS and NTT_CT data types are different over different schemes. One 32-bit accelerator for NTT_GS and another 32-bit for NTT_CT are designed that can support calculation of forward and inverse number theoretic transform over the analyzed schemes with various data bitwidth, which is introduced in this work for the first time.

\section{EVALUATION}

The first step is to identify compute-intensive kernels, Section 4, and then search the space of the design and select the desired design points and evaluate them within an SoC. We use the original software implementation of the accelerated algorithms as a baseline for our evaluation. These implementations are the references for generating the KAT files, which enables us to use them 
Table 1. Size of Loop and Data Types for NTT_GS and NNT_CT over Different Schemes

\begin{tabular}{|l|c|c|c|}
\hline & Loop & \multicolumn{2}{|c|}{ Data type } \\
\cline { 3 - 4 } Scheme & Iterations & Coeffcients & Twiddle factor \\
\hline NewHope512cpa/cca & 256 & uint16_t & uint16_t \\
\hline NewHope1024cpa/cca & 512 & uint16_t & uint16_t \\
\hline Kyber & 128 & uint16_t & uint16_t \\
\hline Dilithium & 128 & uint32_t & uint32_t \\
\hline R.EMBLEM & 512 & int32_t & int32_t \\
\hline AKCN_MLWE & 128 & int32_t & int16_t \\
\hline
\end{tabular}

It should be mentioned that the data type for Keccak perform operations over uint64_t for al the schemes.
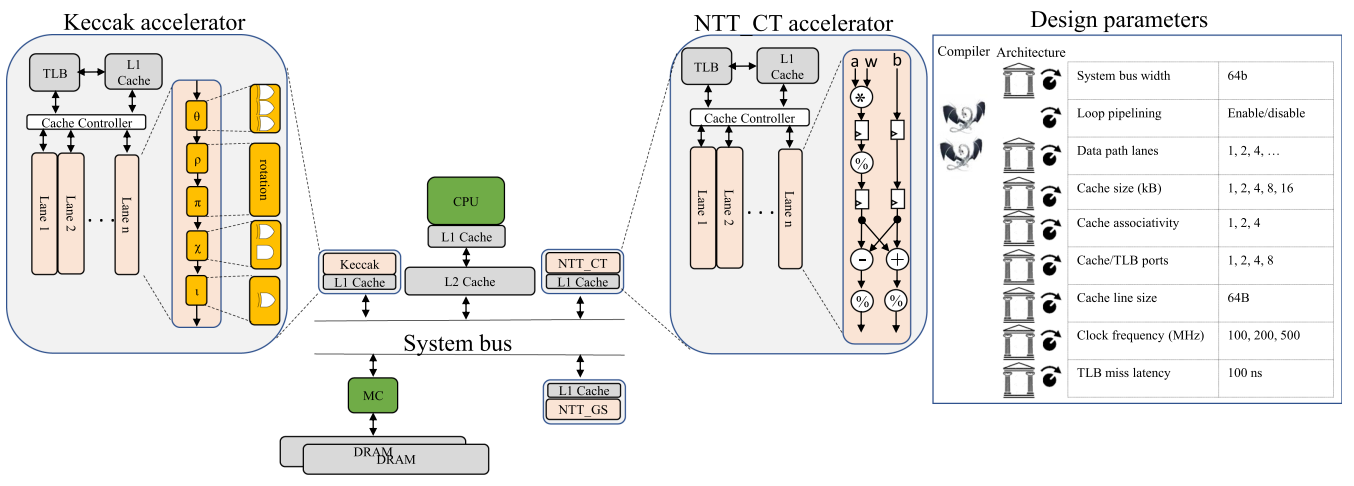

Fig. 7. Architectural template for generated accelerators for Keccak, (NTT_CT), and (NTT_GS) kernels. Sweepable micro-architectural and compiler parameters are shown in the first column of the table; the next column is the searched spaced in this work.

for verifying the accelerator's functionality. To apply our methodology in algorithms, the usage of KAT files can be integrated into the tool flow.

Figure 7 shows the architectural template of the designed accelerators, which are composed of a set of lanes that implement the primitives of the kernel, a Translation Lookaside Buffer (TLB), one level of cache, and the respective controller. Our tool allows analyzing the accelerators without digging into the details of the hardware implementations concentrating on the accelerator functionality.

Based on the profiling results (Figures 2-6), the top three common kernels that consume most of the cycles are NTT_CT, NTT_GS, and Keccak.

Keccak permutation consists of bitwise arithmetic operations, and each datapath lane of the accelerator has basic components to compute the $\mathrm{F}[1600]$ permutation function. The designed accelerator for Keccak can be used in any cryptographic scheme to hash a message and generate pseudo-random numbers. NTT_CT and NTT_GS accelerators are two programmable accelerators that can be employed inside any scheme with arbitrary security parameters to perform the number theoretic transform. Each datapath lane in NTT_CT and NTT_GS accelerators (Figure 7) executes the butterfly operation. To perform NTT and NTT ${ }^{-1}$ in NewHope, NTT_GS is used. However, AKCN_MLWE uses NTT_CT to compute both NTT and NTT ${ }^{-1}$. Kyber, Dilithium, and R.EMBLEM perform NTT with NTT_CT and NTT $^{-1}$ with NTT_GS. 

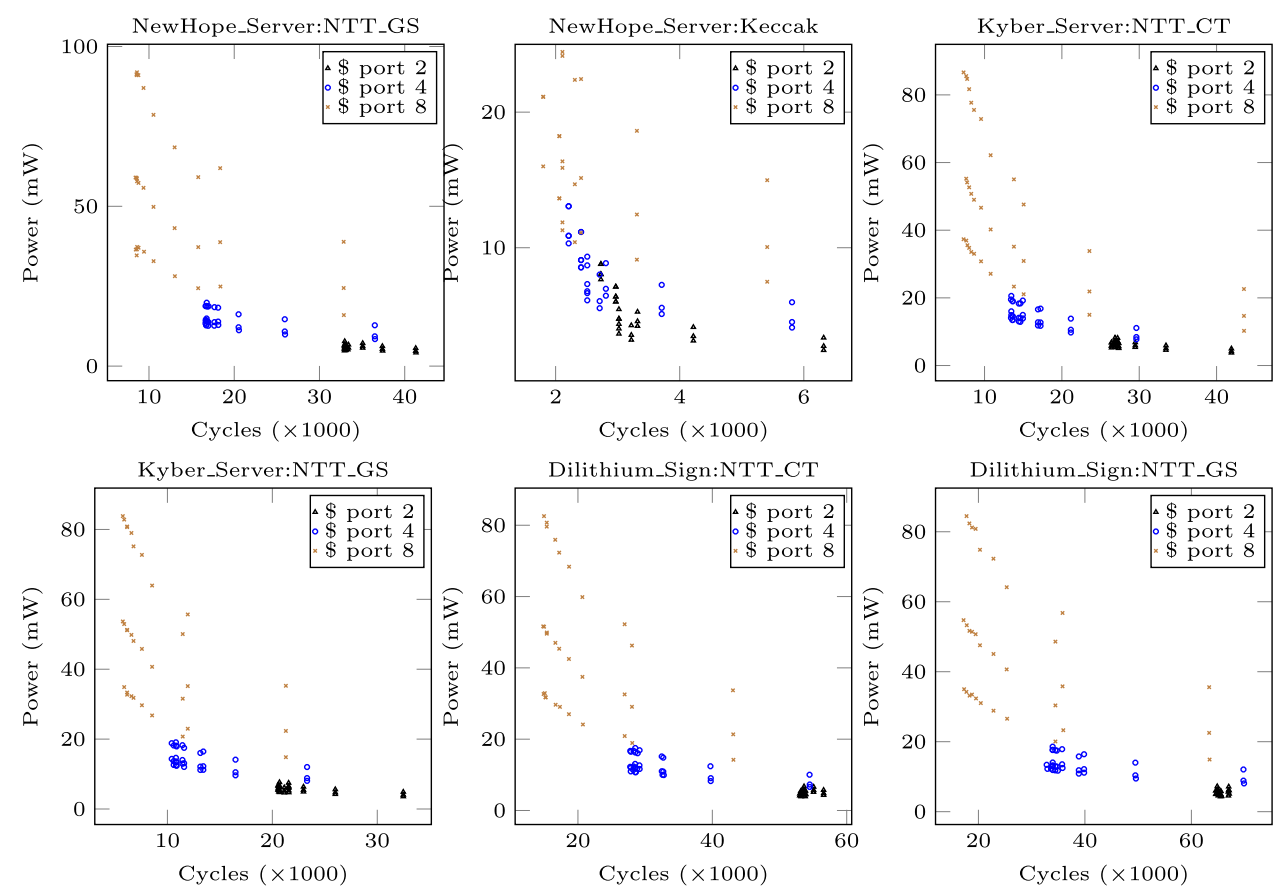

Fig. 8. The sensitivity of the accelerators' performance and power to the number of cache ports. To investigate the sensitivity of the accelerator's power and performance to the number of cache ports, we set number of ports to 1 and sweep all other parameters; afterward, design points that improve the overall performance of the system are extracted. We perform similar sensitivity analysis for cache port sizes of 2, 4, and 8 . For instance, all the green points are the designs with the cache pDeort size of 8 . Similarly, sensitivity analysis for other design parameters is performed. Due to the symmetry of the trends for counterparts in each scheme, we only present the results for the Server and Signer. DSE trends over the schemes are similar; hence, we just present DSE results of NTT_CT for Kyber and NTT_GS for NewHope and Dilithium.

Sensitivity analysis. SoC components share the same system bus width and cache line size; although these two are sweepable parameters, we fixed them at 8 and 64 bytes, since they are the typical sizes of CPU and system bus. The clock frequency of the host processor and system bus are fixed at $2 \mathrm{GHz}$ and $1 \mathrm{GHz}$, respectively. The frequency of the accelerator can be set to 100, 200, or $500 \mathrm{MHz}$.

Figures 8-11 shows the effect of number of cache ports (Figure 8), software pipelining (Figure 9), number of parallel lanes (Figure 10), and accelerator's cache size (Figure 11) on the power and latency of an accelerator in a scheme. Loop pipelining overlaps execution of instructions over different loop iterations by exploiting the loop level parallelism and can be beneficial to improve the performance but increases the hardware complexity and the energy dissipation. Software pipelining is more effective if it is being used with loop unrolling. Loop unrolling determines the number of parallel lanes that are created inside the accelerator for each loop. Loop unrolling improves the performance by alleviating the branch penalty and memory latency with an increase in the code size and the hardware area. Memory reduction latency needs parallel access to memory banks from the lanes, which can be achieved by increasing the number of cache ports; this also incurs high power consumption and area overhead.

The DSE trends for each counterpart in a scheme are similar; hence we only present the results for the Server and the Signer Also, due to the resemblance of the DSE trends, e.g., Kyber_Server: 

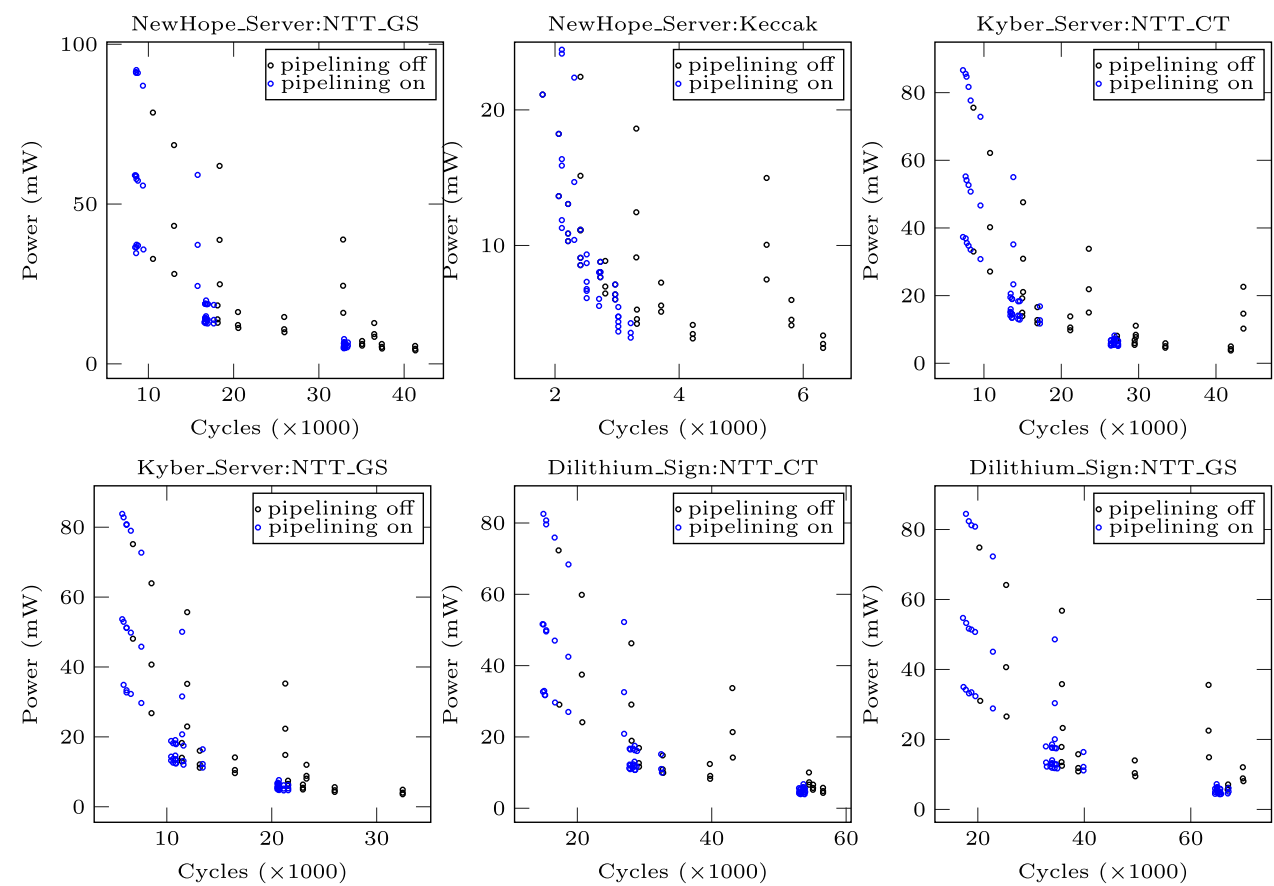

Fig. 9. The sensitivity of the accelerators' performance and power to the software pipelining. Software pipelining is either enabled or disabled.
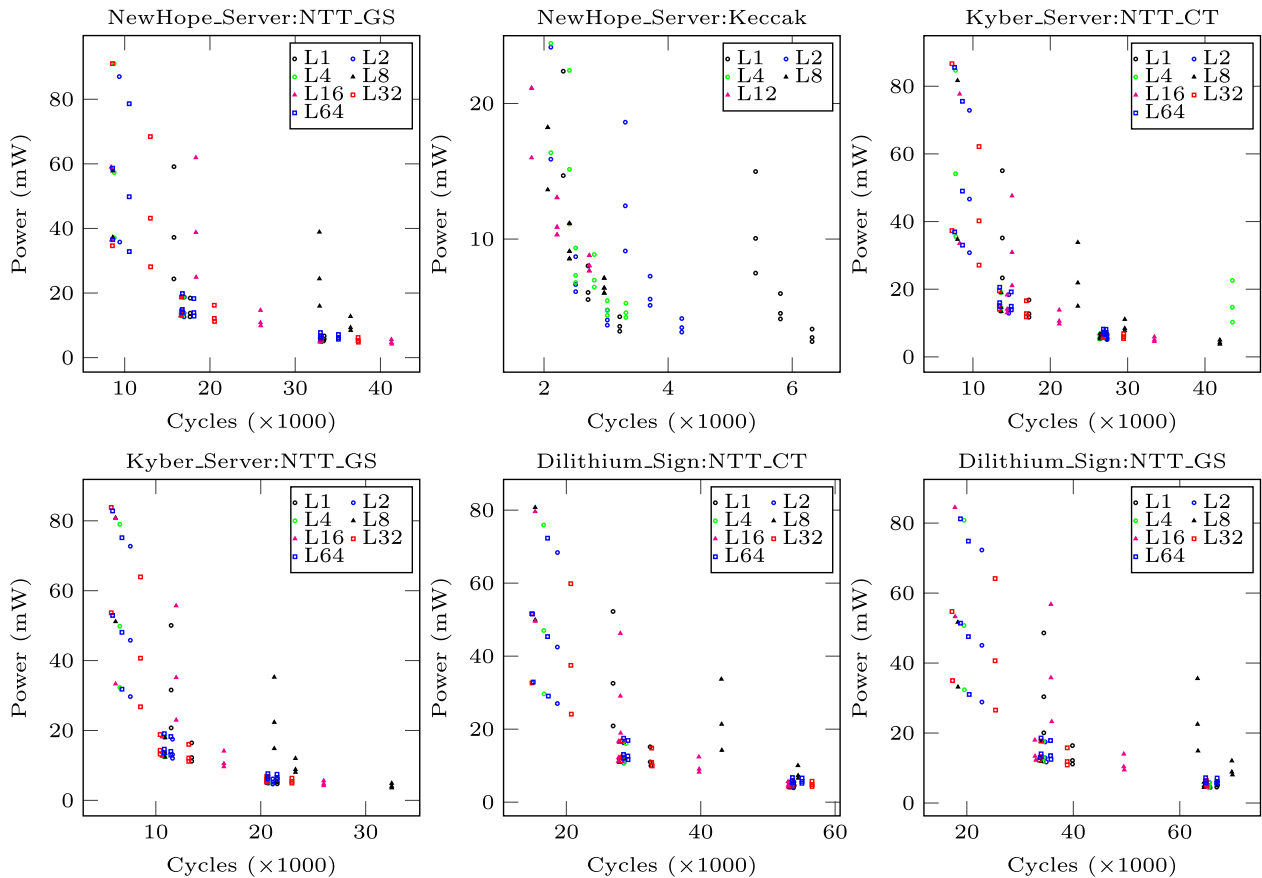

Fig. 10. The sensitivity of the accelerators' performance and power to the number of lanes. Parallel lanes are swept in the range [1-12] for Keccak-f [1600] and [1-64] for NTT_CT and NTT_GS. 

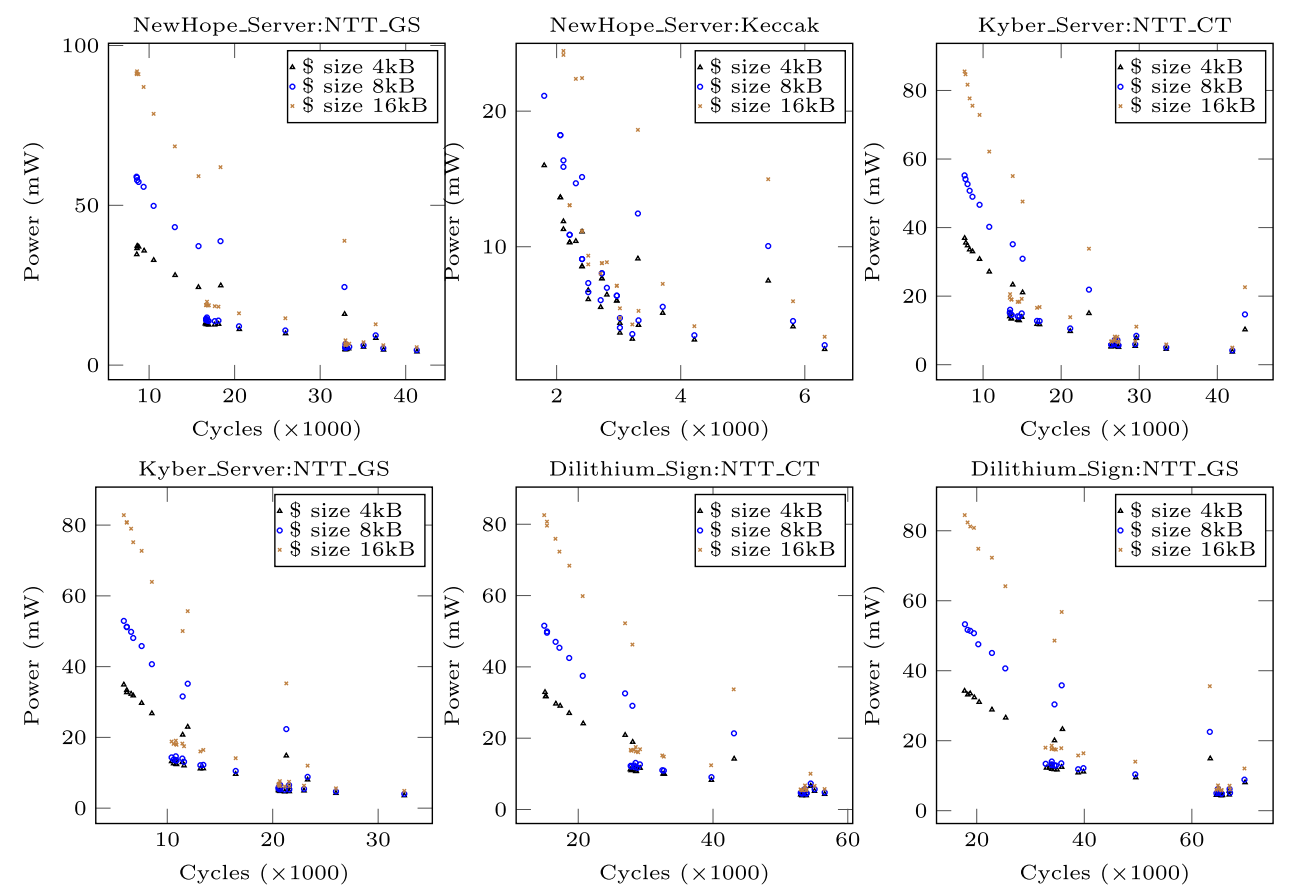

Fig. 11. The sensitivity of the accelerators' performance and power to the cache size. Size of the accelerator's cache is swept in the range $[4 \mathrm{kB}-16 \mathrm{kB}]$ for all the accelerators.

NTT_CT and Dilithium_Sign:NTT_CT, we just present DSE results of NTT_CT for Kyber and NTT_GS for Dilithium.

Selected design points. We carried out our selection focusing first on energy consumption, then the area occupation, and, finally, the speed of each accelerator. Table 2 provides the improvement of the latency, energy, and EDP of the schemes when only one kernel is accelerated, and the rest of the scheme is executed on the CPU. After selecting the desired design points for each accelerator, they are coupled to the bus and communicate via the coherent memory hierarchy (Figure 7). Figure 12 summarizes speedup and EDP improvements of the schemes when all the calls inside of the schemes to Keccak, NTT_CT, and NTT_GS are offloaded to their corresponding optimized accelerators. The frequency of the accelerators 100, 200, and $500 \mathrm{MHz}$.

Based on the results we obtained, we can derive several general guidelines about accelerating lattice-based cryptography. Figure 9 suggests always to enable the loop pipelining, since most of the points on the Pareto-frontier curves are the blue points (pipelining on). The iterative nature of the selected kernels is the main reason for the efficacy of loop pipelining. Considering energy as the main concern, independent of the scheme, after 64 times of loop unrolling the performance gain is saturated for NTT_CT and NTT_GS (Figure 10); the saturation point for Keccak is 12 parallel lanes.

According to Figure 8, the highest speedup is achieved with eight cache ports, considering the performance as the primary concern. However, considering the energy as the primary figure of merit as is our goal, cache port 4 is suggested, because for all the kernels it achieves acceptable performance. Besides, the cache size of $4 \mathrm{kB}$ is proper for all the accelerators, since with bigger cache sizes performance improvements cannot compensate for the energy penalty.

Another consideration is related to the size of the polynomial and the achieved acceleration. The bigger the size of the polynomial, the more speedup we get. NewHope1024 achieves, on average, 
Table 2. Latency and Energy-delay Product (EDP) Improvement of the Schemes (Functional Units Power Models Are Based on OpenPDK 45nm and SRAM Model from CACTI 5.3); One Accelerator (100 MHz) at a Time Is Attached to the Host x86 CPU $(2 \mathrm{GHz})$ through the Bus

\begin{tabular}{|c|c|c|c|c|c|c|c|}
\hline \multirow{2}{*}{ Scheme } & \multirow{2}{*}{ Kernel } & \multicolumn{2}{|c|}{ Cycle Count } & \multirow{2}{*}{$\begin{array}{c}\begin{array}{c}\text { Speedup } \\
(\mathrm{CPU}+\text { Accel })\end{array} \\
\text { Speedup }\end{array}$} & \multicolumn{2}{|c|}{$\begin{array}{l}\text { Improvement } \\
\text { (CPU+Accel) }\end{array}$} & \multirow{2}{*}{$\begin{array}{l}\text { Accelerator's } \\
\text { area }\left(\mathrm{mm}^{2}\right)\end{array}$} \\
\hline & & $\begin{array}{c}\text { CPU } \\
\text { (Baseline) }\end{array}$ & CPU+Accel & & Energy & EDP & \\
\hline \multirow{2}{*}{$\begin{array}{l}\text { NewHope } 1024 \text { cpa } \\
\text { Server }\end{array}$} & NTT_GS & \multirow{2}{*}{1283366} & 676297 & 1.89 & 1.36 & 2.6 & 1.42 \\
\hline & Keccak & & 1282400 & 1.01 & 1.06 & 1.07 & 1.64 \\
\hline \multirow{2}{*}{$\begin{array}{l}\text { NewHope } 1024 \text { cpa } \\
\text { Client }\end{array}$} & NTT_GS & \multirow{2}{*}{1353624} & 741725 & 1.82 & 1.35 & 2.46 & 1.43 \\
\hline & Keccak & & 1352716 & 1 & 1 & 1 & 1.64 \\
\hline \multirow{3}{*}{ Kyber768 Server } & NTT_CT & \multirow{3}{*}{2100227} & 1620437 & 1.29 & 1.11 & 1.44 & 0.42 \\
\hline & NTT_GS & & 1837164 & 1.14 & 1.19 & 1.54 & 1.43 \\
\hline & Keccak & & 2069412 & 1.1 & 1.05 & 1.07 & 1.64 \\
\hline \multirow{3}{*}{ Kyber768 Client } & NTT_CT & \multirow{3}{*}{1090235} & 843838 & 1.29 & 1.19 & 1.54 & 0.42 \\
\hline & NTT_GS & & 951567 & 1.15 & 1.13 & 1.29 & 1.43 \\
\hline & Keccak & & 1052613 & 1.03 & 1.13 & 1.17 & 1.64 \\
\hline \multirow{3}{*}{ Dilithium Sign } & NTT_CT & \multirow{3}{*}{2470672} & 2077058 & 1.18 & 1.07 & 1.45 & 1.36 \\
\hline & NTT_GS & & 1987848 & 1.24 & 1.09 & 1.28 & 1.46 \\
\hline & Keccak & & 2441352 & 1.01 & 1.03 & 1.25 & 1.64 \\
\hline \multirow{3}{*}{ Dilithium Verify } & NTT_CT & \multirow{3}{*}{1278898} & 1060998 & 1.20 & 1.20 & 1.28 & 1.36 \\
\hline & NTT_GS & & 1156888 & 1.10 & 1.16 & 1.35 & 1.46 \\
\hline & Keccak & & 1229706 & 1.04 & 1.20 & 1.05 & 1.64 \\
\hline \multirow{2}{*}{$\begin{array}{l}\text { R.EMBLEM } \\
\text { Server }\end{array}$} & NTT_CT & \multirow{2}{*}{4072534} & 2400767 & 1.7 & 1.5 & 2.5 & 1.36 \\
\hline & NTT_GS & & 3147634 & 1.3 & 1.3 & 1.7 & 1.42 \\
\hline \multirow{2}{*}{$\begin{array}{l}\text { R.EMBLEM } \\
\text { Client }\end{array}$} & NTT_CT & \multirow{2}{*}{1721429} & 1273543 & 1.34 & 1.26 & 1.7 & 1.36 \\
\hline & NTT_GS & & 1101269 & 1.56 & 1.43 & 2.24 & 1.42 \\
\hline $\begin{array}{l}\text { AKCN_LWE } \\
\text { Server }\end{array}$ & NTT_CT & 1216360 & 933151 & 1.3 & 1.2 & 1.56 & 1.36 \\
\hline $\begin{array}{l}\text { AKCN_LWE } \\
\text { Client }\end{array}$ & NTT_CT & 1087624 & 860891 & 1.26 & 1.1 & 1.33 & 1.36 \\
\hline
\end{tabular}

To select a design point and attach the corresponding accelerator to the SoC, we focus first on energy consumption, then the area occupation, and, finally, the speed of each accelerator.

$20 \%$ more speedup than NewHope512 by using the same accelerators, since polynomials in NewHope1024 is twice bigger than NewHope512, and, hence, it gets more benefit from the software pipelining along with loop unrolling. In the case of MLWE schemes, Kyber and Dilithium, size of the polynomials are the same for all different version of the scheme; hence, the speedup for different variants of MLWE schemes is similar.

With the current fast CPU (2 GHz) and energy-efficient accelerator (Figure 12), we suggest to allow the accelerators to operate at the maximum frequency, $500 \mathrm{MHz}$, specifically for Keccak, since at $100 \mathrm{MHz}$ (Table 2) it is not beneficial to use the accelerator. The power consumption of the accelerators is 2 orders of magnitude less than that of the host CPU; consequently, faster accelerators result in better EDP improvement and higher speedup.

Comparing our acceleration with state of the art is challenging, since the acceleration of these algorithms was not presented in literature before. We can, however, compare the achieved performance with results used with the same tool-chain in other domains.

Previous hardware implementations and accelerators. Hardware implementations for the analyzed algorithms are rather scarce, but proponents of R.EMBLEM designed and implemented in the Zynq-7000 board the algorithm EMBLEM.CPA (meaning without the ring structure) [32]. 


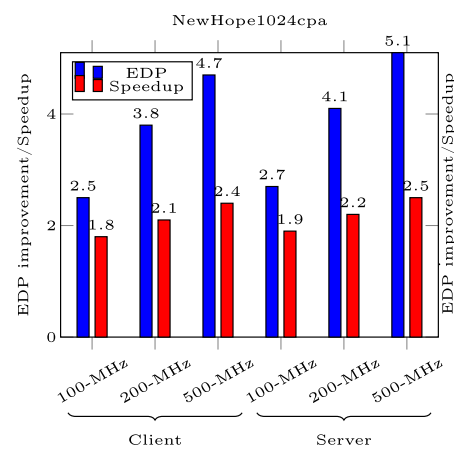

(a) NewHope1024cpa

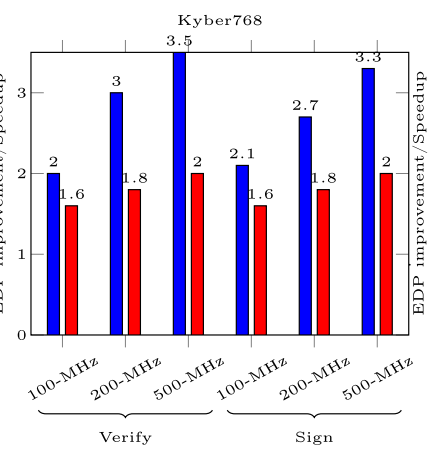

(b) Kyber768

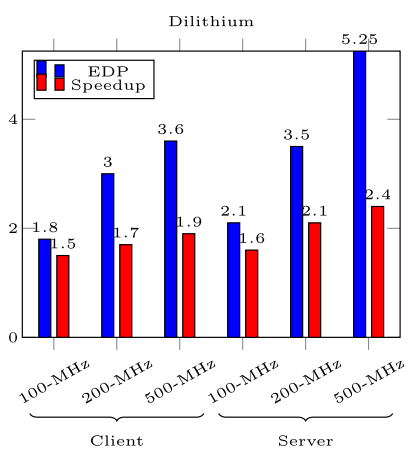

(c) Dilithium

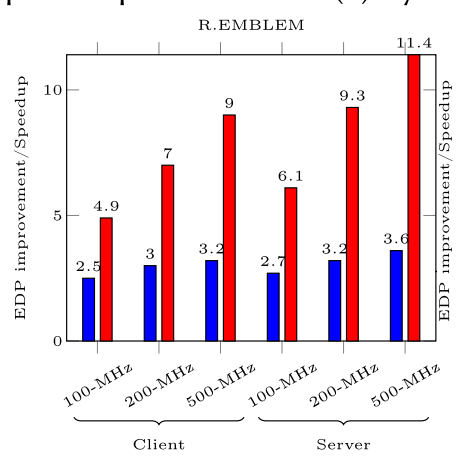

(d) R.EMBLEM

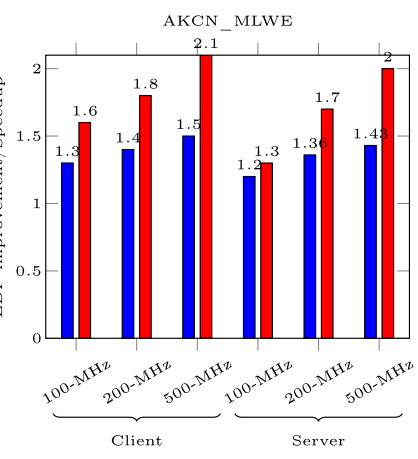

(e) AKCN_MLWE

Fig. 12. Delay and EDP improvement of the schemes when all the accelerators (fastest design points) at different frequencies are simultaneously attached to the bus.

Their design only used 24 FFs and 48 LUTs, but this result can be misleading for evaluating the area of R.EMBLEM.CCA. The same board was used to implement NewHope for high performance. Four butterfly units were employed that use 6,680 slices, 9,412 FFs, 18,756 LUTs, 8DSP, and 14 BRAMS; the implementation took 51.9, 78.6, and $21.1 \mu \mathrm{s}$ for the three phases of NewHope at a frequency of $131 \mathrm{MHz}$ [18]. An existing co-processor for RSA/ECC was repurposed for (ideal) lattice-based cryptography. CCA-secure Kyber768 key generation was executed in 79.6 ms, encapsulation in $102.4 \mathrm{~ms}$, and decapsulation in $132.7 \mathrm{~ms}$ [1]. Aside from the acceleration, this work confirmed that $\mathrm{PQC}$ is feasible on current smart card platforms.

Kanad Basu et al. [7] compared different hardware implementations of PQC algorithms. They used Xilinx HLS-based design space exploration to map C-code to FPGA and ASIC. Differing from us, they created an accelerator with the complete algorithms; instead, we create accelerators for the bottleneck functions. Therefore, we include in our methodology a profiling step to identify the most time-consuming functions, and, hence, our accelerator implements kernels that can be used in different algorithms that result in smaller accelerator (even after a linear correction on the transistor length of the libraries). Additionally, we present the effect on energy, which is an overlooked metric in previous works.

\section{CONCLUSION}

In this article, we concentrate on hardware acceleration for lattice-based algorithms. Since at this stage, it is impossible to commit to a single algorithm, despite the fact that several applications 
would require performance not achievable by the software-only implementation, we propose, for the first time to the best of our knowledge, to design cache-based accelerators that are programmable at two dimensions (kernel sizes data types) for hardware kernels. To this end, we presented a design flow, and we demonstrated its suitability for accelerating NewHope, Kyber, Dilithium, KCL, and R.EMBLEM five submissions to the NIST standardization contest. Our experiments demonstrated the suitability of the approach for exploring the design space of hardware accelerator for lattice-based algorithms, allowing us to quickly identify hardware kernels capable of reducing the energy consumption up to $2.1 \times$ and EDP up to $5.2 \times$ and improve the speedup up to $2.5 \times$. Thanks to the quick exploration and performance estimation, we discover compelling guidelines for manually designing more optimized hardware accelerators for lattice-based algorithms. In the near future, we plan to extend our evaluation to evaluate more lattice-based algorithms and other families of the post-quantum schemes.

\section{REFERENCES}

[1] M. R. Albrecht et al. 2018. Implementing RLWE-based Schemes Using an RSA Co-Processor. Cryptology ePrint Archive, Report 2018/425. Retrieved from https://eprint.iacr.org/2018/425.

[2] E. Alkim et al. 2016. NewHope Without Reconciliation. Cryptology ePrint Archive, Report 2016/1157.

[3] S. A. Ansarmohammadi et al. 2015. Fast and area efficient implementation for chaotic image encryption algorithms. In Proceedings of the 2015 18th CSI International Symposium on Computer Architecture and Digital Systems (CADS'15).

[4] R. Avanzi et al. 2017. CRYSTALS-KYBER. Technical Report. NIST.

[5] T. Bai et al. 2014. Analysis and acceleration of NTRU lattice-based cryptographic system. In Proceedings of the IEEE/ACIS International Conference on Software Engineering, Artificial Intelligence, Networking and Parallel/Distributed Computing (SNPD'14).

[6] U. Banerjee et al. 2019. Sapphire: A configurable crypto-processor for Post-QuantumLattice-based protocols. IACR Trans. Cryptogr. Hardw. Embed. Syst. (2019).

[7] K. Basu et al. 2019. NIST Post-Quantum Cryptography- A Hardware Evaluation Study. Cryptology ePrint Archive, Report 2019/047.

[8] G. Bertoni et al. 2011. The Keccak reference.

[9] N. Binkert et al. 2011. The Gem5 simulator. SIGARCH(2011).

[10] J. Cooley et al. 1965. An algorithm for the machine calculation of complex fourier series. Math. Comp. (1965).

[11] M. R. Doomun et al. 2007. Energy consumption and computational analysis of rijndael-AES. In Proceedings of the International Conference in Central Asia on Internet.

[12] L. Ducas et al. 2017. CRYSTALS-Dilithium. Technical Report. National Institute of Standards and Technology.

[13] M. Dworkin. 2015. SHA-3 Standard: Permutation-based Hash and Extendable-output Functions. Technical Report.

[14] R. P. Feynman. 1982. Simulating physics with computers. Int. F. Theor. Phys. (1982).

[15] E. Fujisaki et al. 2013. Secure integration of asymmetric and symmetric encryption schemes. F. Cryptol. (2013).

[16] W. M. Gentleman et al. 1966. Fast fourier transforms: For fun and profit. In Proceedings of the Annual Confernece of the American Federation of Information Processing Societies (AFIPS'66).

[17] T. Guneysu et al. 2017. Towards lightweight identity-based encryption for the post-quantum-secure Internet of Things. In Proceedings of the International Symposium on Quality Electronic Design (ISQED'17).

[18] P. Kuo et al. 2017. High Performance Post-Quantum Key Exchange on FPGAs. Cryptology ePrint Archive, Report 2017/690. Retrieved from https://eprint.iacr.org/2017/690.

[19] A. Langlois et al. 2012. Worst-Case to Average-Case Reductions for Module Lattices. Cryptology ePrint Archive.

[20] V. Lyubashevsky. 2009. Fiat-Shamir with aborts: Applications to lattice and factoring-based signatures. In Proceedings of the Annual International Conference on the Theory and Application of Cryptology and Information Security (ASIACRYPT'09).

[21] V. Lyubashevsky et al. 2010. On Ideal Lattices and Learning with Errors over Rings. In Proceedings of the International Conference on the Theory and Applications of Cryptographic Techniques (EUROCRYPT'10).

[22] M. Naehrig et al. 2017. FrodoKEM. Technical Report. National Institute of Standards and Technology.

[23] H. Nejatollahi et al. 2017. Trends, challenges and needs for lattice-based cryptography implementations: Special session. In Proceedings of the (CODES'17).

[24] H. Nejatollahi et al. 2018. Domain-specific Accelerators for Ideal Lattice-based Public Key Protocols. Cryptology ePrint Archive, Report 2018/608. 
[25] H. Nejatollahi et al. 2019. Exploring Energy Efficient Quantum-resistant Signal Processing Using Array Processors. Cryptology ePrint Archive.

[26] H. Nejatollahi et al. 2019. Flexible NTT accelerators for RLWE lattice-based cryptography. Proceedings of the IEEE International Conference on Computer Design (ICCD'19).

[27] H. Nejatollahi et al. 2019. Post-quantum lattice-based cryptography implementations: A survey. ACM Comput. Surv. (2019).

[28] NTTCorporation. 2008. PSEC-KEM Specification.

[29] J. Olson et al. 2017. Quantum information and computation for chemistry. arXiv preprint arXiv:1706.05413 (2017).

[30] T. Poppelmann et al. 2017. NewHope. Technical Report. NIST.

[31] O. Regev. 2005. On lattices, learning with errors, random linear codes, and cryptography. (2005).

[32] M. Seo et al. 2017. EMBLEM and R.EMBLEM. Technical Report. National Institute of Standards and Technology.

[33] S. Shao et al. 2016. Co-designing accelerators and soc interfaces using gem5-aladdin. In Proceedings of the IEEE/ACM International Symposium on Microarchitecture (MICRO'16).

[34] P. Shor. 1997. Polynomial-time algorithms for prime factorization and discrete logarithms on a quantum computer. Proceedings of the Annual International SICOMP Conference (SICOMP'97).

[35] E. E. Targhi et al. 2016. Post-quantum security of the Fujisaki-Okamoto and OAEP transforms. In Theory of Cryptography.

[36] J. Toldinas et al. 2011. Energy efficiency comparison with cipher strength of AES and Rijndael cryptographic algorithms in mobile devices. (2011).

[37] A. S. Wander et al. 2005. Energy analysis of public-key cryptography for wireless sensor networks. In Proceedings of the IEEE International Conference on Pervasive Computing and Communications.

[38] Y. Zhao et al. 2017. A Modular and Systematic Approach to Key Establishment and Public-Key Encryption Based on LWE and Its Variants. Technical Report. National Institute of Standards and Technology. Retrieved from https://csrc.nist. gov/projects/post-quantum-cryptography/round-1-submissions.

Received March 2019; revised August 2019; accepted January 2020 